\title{
HYBRID OPTIMIZATION METHOD FOR COGNITIVE AND MIMO RADAR CODE DESIGN
}

\author{
Tuomas Aittomäki and Visa Koivunen \\ Department of Signal Processing and Acoustics, \\ Aalto University \\ PO Box 13000, FI-00076 Aalto, Finland \\ Email: tuomas.aittomaki@aalto.fi, visa.koivunen@aalto.fi
}

\begin{abstract}
Cognitive and MIMO radars need to adapt the transmitted waveforms based on the radar task as well as the propagation and the target environments. Many waveform optimization methods proposed in the literature for optimizing the sidelobe and cross-correlation levels are based on stochastic search algorithms or slow numerical approximation methods. However, for real-time applications, it is necessary to perform the optimization fast since the radar channels and target parameters may vary rapidly. For this purpose, we propose a hybrid optimization approaches based on gradient and randomization for fast optimization of the transmit waveform codes.
\end{abstract}

Index Terms - Cognitive radar, MIMO radar, waveform design, optimization, constant-modulus waveforms

\section{INTRODUCTION}

Cognitive radars are intelligent radar systems that are aware of their surroundings and use the received information for learning and adaptation of its operation [1]. MIMO radars, on the other hand, are defined as a radar systems that transmit simultaneously from multiple transmitters and receive the scattered signal using multiple receivers $[2,3]$. Furthermore, MIMO radars use feedback from the receivers to the transmitters for cooperative transmission, including optimization for a particular target scenario and radar task [4].

Typical goal for waveform optimization is to achieve low peak sidelobe and cross-correlation levels, and the optimization of the symbols for the transmitted radar waveforms has been studied exetnsively in the past. For example, simulated annealing and iterative code selection were combined to search for orthogonal polyphase codes in [5]. Similar approach using genetic algorithm was used in [6]. Tabu search algorithm was used in [7] and the cross-entropy method in [8]. An effective optimization method was introduced in [9]. While there are simple solutions for waveforms with optimal SINR, for example (see [10] or [11]), no such solutions have been presented for the general case of optimizing sidelobe and cross-correlation levels.

The optimization techniques employed in these methods have high complexity and may not be applicable in scenarios with rapidly varying propagation conditions and target parameters. In such situations, the sidelobe and cross-correlation levels need to be adjusted according to the returned signal power in order to keep the probability of errors constant. Therefore, it is necessary to be able to do the code design for cognitive and MIMO radars in real-time.

Many of the existing optimizations in the literature, such as those cited above, require large number of iterations to provide good re-

This work was supported by the Finnish Defence Research Agency. sults. Newton-type algorithms can be used for faster convergence, but the problem with this approach is that when optimizing the peak sidelobe and cross-correlation levels, the objective is highly nonconvex. Thus, conventional gradient-based methods typically get stuck in the first encountered local minimum with suboptimal, typically even poor results.

In this paper, we propose a hybrid method that uses randomization to alleviate the problems of the gradient-based algorithms. Whenever the gradient-based method cannot decrease the cost, a search direction is chosen randomly forming a hybrid of gradientbased and stochastic search methods. This approach can be applied to the gradient descent, the conjugate gradient, and the BroydenFletched-Goldfarb-Shanno (BFGS) method, for example. Points at which the objective function is not differentiable either a subgradient or a random direction can be used. It should be noted that this approach is entirely different from the stochastic gradient descend.

In this paper, we develop a waveform code design method that combines a randomized approach with the conjugate gradient on the oblique manifold. Furthermore, by choosing the right parametrization, the manifold constraints of the code design problem can be converted into simple linear constraints enabling the use of the BFGS method. These methods are compared with simulated annealing. It is demonstrated that these hybrid methods achieve far better results than the conventional gradient based approaches. The hybrid conjugate gradient approach results in similar peak sidelobe and crosscorrelation levels as the simulated annealing, but with an order of magnitude smaller computational complexity.

The paper is orgnaized as follows. The problem Formulation is given in Section 2. In Section 3, we describe the proposed optimization approaches. Numerical examples of the optimization results are given in Section 4, before the final conclusions in Section 5.

\section{PROBLEM FORMULATION}

The goal is to design the code sequences so that the coded waveforms would have low peak sidelobe and peak cross-correlation levels. The cross-ambiguity function is defined for narrowband waveforms $s_{i}(t)$ and $s_{j}(t)$ as [12]

$$
\chi_{i j}\left(\tau, F_{D}\right)=\left|\int s_{i}(t) s_{j}^{*}(t+\tau) e^{\mathrm{j} 2 \pi F_{D} t} d t\right|^{2},
$$

where $\tau$ is the time delay, $F_{D}$ is the Doppler frequency of the target, and $\mathfrak{j}$ is the imaginary unit. If the narrowband assumption is not valid, the cross-ambiguity function has to be written as [13]

$$
\chi_{i j}\left(\tau, F_{D}\right)=\left|\sqrt{\gamma} \int s_{i}(t) s_{j}^{*}(\gamma t+\tau) e^{\mathrm{j} 2 \pi F_{D} t} d t\right|^{2} .
$$


The temporal compression factor $\gamma$, which describes how much the waveform compresses or stretched in time because of the Doppler shift, is given by

$$
\gamma=1+\frac{F_{D}}{F_{c}}
$$

where $F_{c}$ is the carrier frequency.

As most radars use digital signal processing, we are interested in the properties of sampled discrete-time signals. Therefore, we define the sample cross-ambiguity function as

$$
\chi_{i j}\left(\tau, F_{D}, T_{s}\right)=\left|T_{s} \sqrt{\gamma} \sum_{k} s_{i}\left(k T_{s}\right) s_{j}^{*}\left(\gamma k T_{s}+\tau\right) e^{\mathrm{j} 2 \pi F_{D} k T_{s}}\right|^{2},
$$

where $T_{s}$ is the sampling interval. Using the definition of Riemann integral, we see that as $T_{s} \rightarrow 0$, the sample cross-ambiguity function converges to the continuous-time one if (2) is integrable.

The peak cross-correlation (PCC) can be used as a measure of the maximum of the sample cross-ambiguity function. The normalized PCC is defined for the $i$ th waveform as

$$
\operatorname{PCC}_{i}\left(T_{s}\right)=\sup _{j, \tau, F} \frac{\chi_{i j}\left(\tau, F, T_{s}\right)}{\chi_{i i}\left(0,0, T_{s}\right)} .
$$

The normalized peak sidelobe (PSL) of the sample auto-ambiguity function is defined as

$$
\operatorname{PSL}_{i}\left(T_{s}\right)=\sup _{(\tau, F) \notin \mathcal{P}_{i}} \frac{\chi_{i i}\left(\tau, F, T_{s}\right)}{\chi_{i i}\left(0,0, T_{s}\right)},
$$

where the set $\mathcal{P}_{i}$ is defined as the set of delay $\tau$ and Doppler frequency $F$ values that encompass the main peak of the $i$ th waveform. Typically, $F$ and $\tau$ are continuous but can be discretized in suitable way as discussed below.

Let the vector $\mathbf{s}_{i}$ contain the samples of the $i$ th baseband waveform $s_{i}(t)$, i.e. $\left(\mathbf{s}_{i}\right)_{n}=s_{k}\left(n T_{s}\right)$, where $T_{s}$ is the sampling interval The dimensions of $\mathbf{s}_{k}$ depend on the number of symbols and sampling rate, and at critical sampling rate, the $s_{i}$ would be a $N_{p} \times 1 \mathrm{vec}-$ tor, where $N_{p}$ is the number of pulses, which is equal to the number of symbols. We define the delay and Doppler matrix $\mathbf{D}\left(\tau, F_{D}, T_{s}\right)$ as

$$
\left(\mathbf{D}\left(\tau, F_{D}, T_{s}\right)\right)_{n, m}=\sqrt{\gamma} T_{s} e^{\mathrm{j} 2 \pi n F_{D} T_{s}} \delta_{h(n), m},
$$

where $\gamma$ is given by (3), $\delta_{i j}$ is the Kronecker delta, and

$$
h(n)=\left\lfloor\frac{n T_{s}+\tau}{\gamma T_{s}}\right\rfloor .
$$

Thus, D has nonzero elements only on one of the diagonals (not necessarily the main one) depending on the delay $\tau$.

Using the delay and Doppler matrix, the sample cross-ambiguity function can then be written as

$$
\chi_{i j}\left(\tau, F_{D}, T_{s}\right)=\left|\mathbf{s}_{i}^{H} \mathbf{D}\left(\tau, F_{D}, T_{s}\right) \mathbf{s}_{j}\right|^{2} .
$$

In order to simplify the waveform optimization problem, it is assumed next that the narrowband assumption holds, and that the waveform is sampled so that the symbol duration is an integer multiple of sampling interval $T_{s}$. With these assumptions, only the sample index $k$ and the normalized Doppler frequency $f$ are needed for forming the delay and Doppler matrix. If the waveform is critically sampled (the sampling rate is equal to the symbol rate), the delay and Doppler matrix in (7) becomes a $N_{p} \times N_{p}$ matrix with elements

$$
\begin{aligned}
\left(\mathbf{D}_{k, f}\right)_{n, m} & =\left(\mathbf{D}\left(k T_{s}, F_{D}, T_{s}\right)\right)_{n, m} \\
& =T_{s} e^{j 2 \pi n F_{D} T_{s}} \delta_{n+k, m}=T_{s} e^{j 2 \pi f n} \delta_{n+k, m},
\end{aligned}
$$

where normalized Doppler frequency is $f=F_{D} T_{s}$.

We can also assume without loss of generality that each waveform is normalized such that

$$
\left\|s_{k}\right\|^{2}=1
$$

The PSL and the PCC of the $i$ th waveform can now be expressed as

$$
\begin{aligned}
\mathrm{PSL}_{i} & =\sup _{(k, f) \notin \mathcal{P}_{i}}\left|\mathbf{s}_{i}^{H} \mathbf{D}_{k, f} \mathbf{s}_{i}\right|^{2}, \\
\mathrm{PCC}_{i} & =\sup _{j, k, f}\left|\mathbf{s}_{i}^{H} \mathbf{D}_{k, f} \mathbf{s}_{j}\right|^{2} .
\end{aligned}
$$

Assuming that the half-width of the main peak is one symbol in the delay and $f_{0}$ in the normalized Doppler frequency, the condition $(k, f) \notin \mathcal{P}_{i}$ can be written as $|f| \geq \delta_{k 0} f_{0}$, where $\delta_{i j}$ is the Kronecker delta.

The goal of minimizing the maximum PSL and PCC of the waveform set can now be formulated as an optimization problem

$$
\begin{array}{ll}
\min _{\mathbf{s}_{i}, \mathbf{s}_{j}} \max _{i, j, k, f}\left|\mathbf{s}_{i}^{H} \mathbf{D}_{k, f} \mathbf{s}_{j}\right|^{2} & |f| \geq \delta_{i j} \delta_{k 0} f_{0} \\
\text { s.t. }\left\|\mathbf{s}_{i}\right\|^{2}=1, & \forall i .
\end{array}
$$

The function $\left|\mathbf{s}_{i}^{H} \mathbf{D}_{k, f} \mathbf{s}_{j}\right|^{2}$ is a continuous, multivariate polynomial and $k$ is integer whereas $f \in[-1 / 2,1 / 2]$ due to the unit-period of the ambiguity function and aliasing. Consequently, the maximum exists. By discretizing the normalized Doppler frequency, one obtains a minimax optimization problem of multivariate quartic polynomials on an oblique manifold.

\section{OPTIMIZATION METHODS}

In order to use gradient-based approaches for the code design of the transmit waveform, one needs the gradient of the objective function (15a). We concatenate the symbol vectors into a single vector as

$$
\mathbf{s}=\left(\begin{array}{llll}
\mathbf{s}_{1}^{T} & \mathbf{s}_{2}^{T} & \ldots & \mathbf{s}_{M}^{T}
\end{array}\right)^{T},
$$

where $M$ is the number of waveforms.

Let $\mathbf{u}_{i}$ be an unit vector such that

$$
\left(\mathbf{u}_{i}\right)_{k}= \begin{cases}1, & k=i \\ 0, & \text { otherwise }\end{cases}
$$

The waveform selection matrix $\mathbf{C}_{i}$ such that $\mathbf{C}_{i} \mathbf{s}=\mathbf{s}_{i}$ can then be defined as

$$
\mathbf{C}_{i}=\mathbf{u}_{i}^{T} \otimes \mathbf{I}_{M},
$$

where $\otimes$ denotes the Kronecker product and $\mathbf{I}_{M}$ is an $M \times M$ identity matrix. With $\mathbf{A}_{i, j, k, f}=\mathbf{C}_{i}^{T} \mathbf{D}_{k, f} \mathbf{C}_{j}$, the objective function can be written as

$$
\max _{i, j, k, f}\left|\mathbf{s}^{H} \mathbf{A}_{i, j, k, f} \mathbf{s}\right|^{2}
$$

Defining $q_{i, j, k, f}=\mathbf{s}^{H} \mathbf{A}_{i, j, k, f} \mathbf{s}$, the calculation rules of the complex gradient [14] can be used to obtain

$$
\nabla_{\mathbf{s}}\left|q_{i, j, k, f}\right|^{2}=2\left(q_{i, j, k, f}^{*} \mathbf{A}_{i, j, k, f}+q_{i, j, k, f} \mathbf{A}_{i, j, k, f}^{H}\right) \mathbf{s} .
$$

However, the objective function (15a) is not differentiable at points where the values of $i, j, k, f$ are not unique for the maximum. In such points, a subgradient can be obtained by setting the elements of 
the gradient vector to zero whenever the real or the imaginary parts of the gradient vectors corresponding the different sets of $i, j, k, f$ have a different sign.

When a descend direction cannot be found with the conventional gradient approaches (including the conjugate gradient), a random direction can be used as a new search direction. A new direction is drawn from the multivariate normal distribution or the uniform distribution on a complex hypersphere until a direction that decreases the objective function is found (or the maximum allowed number of function evaluations has been reached). Also, uphill climb may initially be allowed with the randomized search directions.

In order to use the BFGS method, we use an alternative parametrization for the symbols so that the manifold constraints are converted into a simpler linear constraints. We parametrize the symbols as

$$
(\mathbf{s})_{n}=\sqrt{(\boldsymbol{\rho})_{n}} e^{\mathbf{j}(\boldsymbol{\phi})_{n}},
$$

where $j$ is the imaginary unit and $\rho$ and $\phi$ are real-valued vectors. With this parametrization, $\left\|\mathbf{s}_{i}\right\|^{2}=\mathbf{1}^{T} \mathbf{C}_{i} \boldsymbol{\rho}=1$ is a simple linear constraint. This constraint is easily satisfied by projecting the search direction. Additionally, a constraint $(\boldsymbol{\rho})_{n} \geq 0 \forall n$ is required, whereas $\phi$ is unconstrained. Therefore, the BFGS with bound constraints can be used. For constant-modulus codes, only the parameter vector $\phi$ is required.

Next, we derive the gradients in the parametrization (21). This time, the ordinary chain rule can be used to obtain

$$
\begin{aligned}
\nabla_{\boldsymbol{\rho}} q_{i, j, k, f} & =\frac{1}{2} \operatorname{diag}\left(\mathbf{A}_{i, j, k, f} \mathbf{Y}+\mathbf{Y}^{H} \mathbf{A}_{i, j, k, f}\right) \\
\nabla_{\boldsymbol{\phi}} q_{i, j, k, f} & =\mathfrak{j} \operatorname{diag}\left(\mathbf{X} \mathbf{A}_{i, j, k, f}-\mathbf{A}_{i, j, k, f} \mathbf{X}\right),
\end{aligned}
$$

where the elements of the matrices $\mathbf{X}$ and $\mathbf{Y}$ are defined as $(\mathbf{X})_{i j}=$ $(\mathbf{s})_{i}(\mathbf{s})_{j}^{*}$ and $(\mathbf{Y})_{i j}=\frac{(\mathbf{s})_{i}}{(\mathbf{s})_{j}}$, respectively. The function $\operatorname{diag}(\cdot)$ is a vector consisting of the diagonal elements of the matrix argument. Using an auxiliary matrix

$$
\mathbf{B}_{i, j, k, f}=q_{i, j, k, f}^{*} \mathbf{A}_{i, j, k, f}+q_{i, j, k, f} \mathbf{A}_{i, j, k, f}^{H},
$$

we finally obtain

$$
\begin{aligned}
\nabla_{\boldsymbol{\rho}}\left|q_{i, j, k, f}\right|^{2} & =\operatorname{Re}\left[\left(\mathbf{B}_{i, j, k, f} \mathbf{s}\right) \oslash \mathbf{s}\right] \\
\nabla_{\phi}\left|q_{i, j, k, f}\right|^{2} & =2 \operatorname{Im}\left[\left(\mathbf{B}_{i, j, k, f} \mathbf{s}\right)^{*} \odot \mathbf{s}\right],
\end{aligned}
$$

where $\oslash$ denotes element-wise division and $\odot$ element-wise multiplication.

\section{NUMERICAL EXAMPLES}

Numerical examples of the proposed optimization approach are provided next. In order to compare the code design methods, we optimized a waveform set consisting of three waveforms with 40 symbols in each. Slow-time coding was assumed, so the normalized Doppler frequency could take any values between $-\frac{1}{2}$ and $\frac{1}{2}$. The objective was to obtain waveforms with lowest possible peak sidelobe and peak cross-correlation levels. The proposed hybrid approach was used with the conjugate gradient (hCG) on the manifold and the hybrid BFGS (hBFGS) using the alternative parametrization. These methods were compared with simulated annealing (SA) as well as particle swarm optimization (PSO), which is a populationbased, stochastic algorithm for optimization of continuous nonlinear functions [15].

The initial guess was chosen randomly, and the each method iterated until 15000 function evaluations had been done. A typical outcome for the optimization can be seen in Fig. 1 showing the

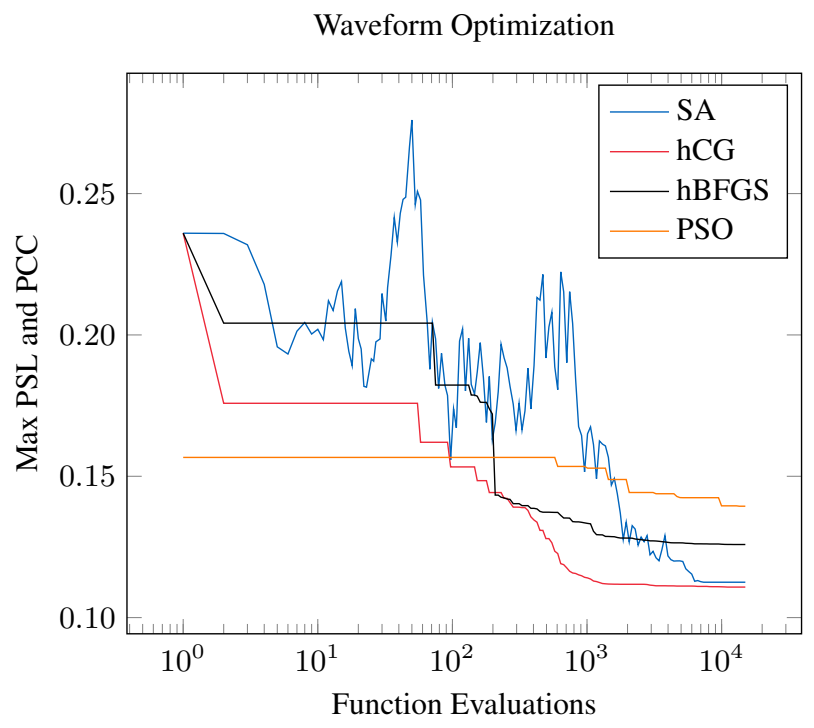

Fig. 1. Achieved PSL and PCC as a function of used function evaluations. SA and the hCG achieve similar PSL and PCC, but the hCG requires only a fraction of the function evaluations.

achieved peak sidelobe level (PSL) and peak cross-corelation (PCC) as a function of used function evaluations. The hybrid conjugate gradient (hCG) and the simulated annealing achieve approximately the same PSL and PCC levels. However, the hCG requires only a thousand function evaluations, whereas the SA requires nearly 10000 for the same result.

As the proposed method is partly stochastic, the outcome varies with each run. This is demonstrated in Fig.2 showing the results for different instance. This time, SA has provided lowest PSL and PCC levels, and also PSO has achieved a better result than the proposed methods.

In order to compare the performance more consistently, the mean and variance of the achieved PSL and PCC were calculated over one thousand runs. The results are shown in Fig. 3 displaying the average PSL and PCC. The averages are marked with the solid lines whereas the dashed lines show one standard deviation. The proposed hCG method performs consistently well with far fewer function evaluations compared to the other methods. On the other hand, the decrease of the PSL and PCC is much lower with the hBFGS and its variance stays large. The PSO has a low initial variance as it has multiple initial guesses, but it would seem to require much larger amount of function evaluation to achieve good waveforms.

\section{CONCLUSIONS}

Cognitive and MIMO radars often need to optimize the transmitted waveforms in real-time because of the rapidly varying state of the radar spectrum and target parameters. In this paper, we proposed a hybrid optimization approach that uses the conjugate gradient and randomization for the search direction in the optimization of the peak sidelobe and cross-correlation levels.

In the numerical examples, the proposed hCG method using conjugate gradient performed consistently well achieving similar results to simulated annealing, but with only a fraction of the function evaluations required. Hence, it is more feasible for real-time waveform optimization. The BFGS algorithm was also tested, but it did not 


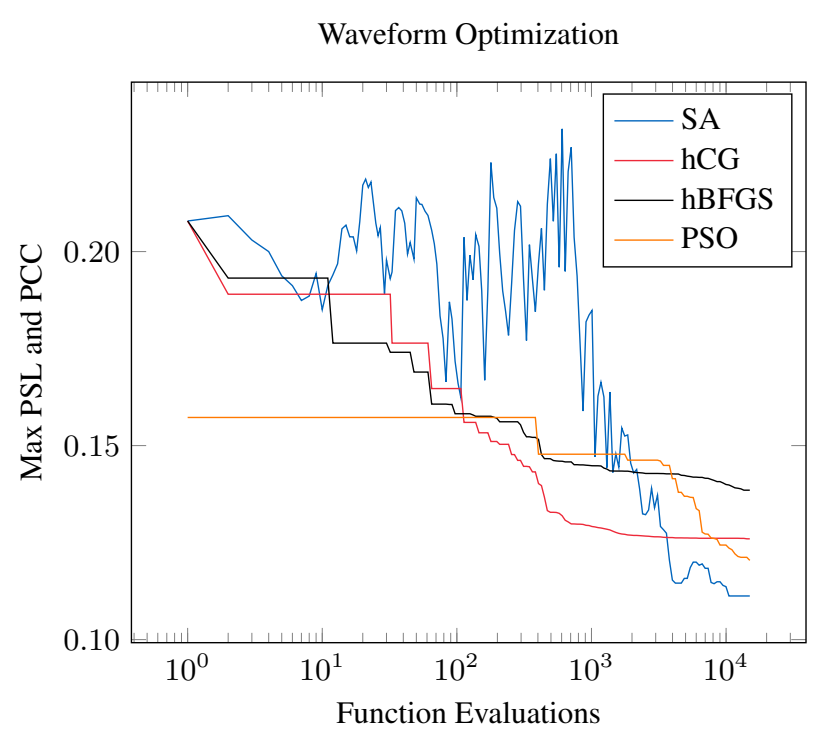

Fig. 2. Alternative outcome of the compared methods. In this case, simulated annealing has performed the best.

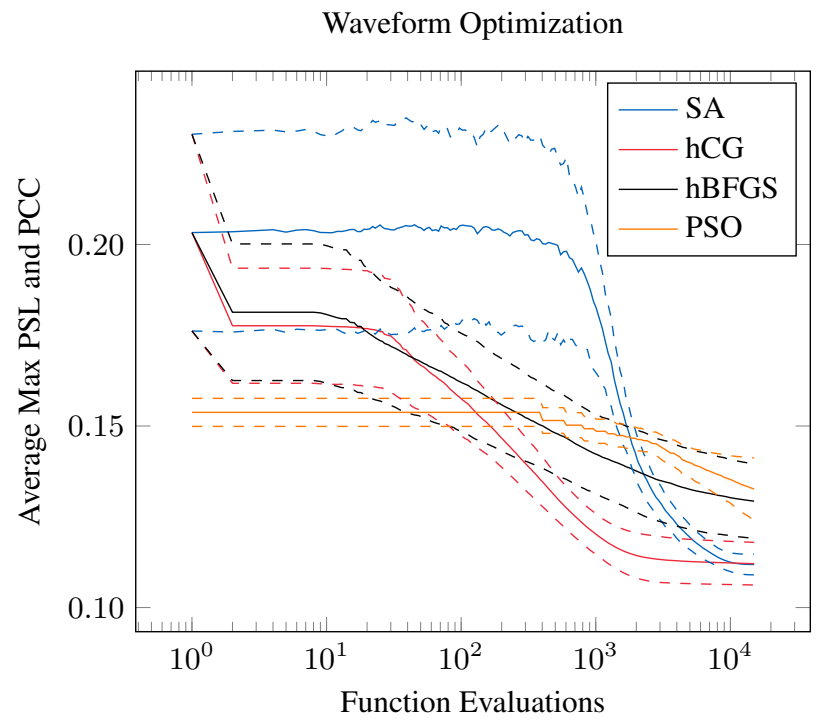

Fig. 3. Mean of the Max PSL and PCC for thousand runs. The dashed lines show plus or minus one standard deviation from the mean. The proposed CG method performs consistently well. perform as well. The conjugate gradient on the manifold using the randomization was found out to be efficient for the given waveform design problem.

\section{REFERENCES}

[1] S. Haykin, "Cognitive radar networks," in 1st IEEE International Workshop on Computational Advances in Multi-Sensor Adaptive Processing, Dec. 2005, pp. 1-3.

[2] Jian Li and Petre Stoica, "MIMO radar with colocated antennas," IEEE Signal Processing Magazine, vol. 24, no. 5, pp. 106-114, Sept. 2007.

[3] A.M. Haimovich, R.S. Blum, and L.J. Cimini, "MIMO radar with widely separated antennas," IEEE Signal Processing Magazine, vol. 25, no. 1, pp. 116-129, Jan. 2008.

[4] A.M. Haimovich, R.S. Blum, and L.J. Cimini, "MIMO radar with widely separated antennas," IEEE Signal Processing Magazine, vol. 25, no. 1, pp. 116-129, Jan. 2008.

[5] Hai Deng, "Polyphase code design for orthogonal netted radar systems," IEEE Transactions on Signal Processing, vol. 52, no. 11, pp. 3126-3135, Nov. 2004.

[6] Bo Liu, Zishu He, Jiankui Zeng, and Benyong Liu, "Polyphase orthogonal code design for MIMO radar systems," in International Conference on Radar, Oct. 2006, pp. 1-4.

[7] Dali Liu, Yuntao Liu, and Huizhi Cai, "Orthogonal polyphase code sets design for MIMO radar using tabu search," in IEEE International Conference on Intelligent Control, Automatic Detection and High-End Equipment, July 2012, pp. 123127.

[8] H.A. Khan, Yangyang Zhang, C. Ji, C.J. Stevens, D.J. Edwards, and D. O'Brien, "Optimizing polyphase sequences for orthogonal netted radar," IEEE Signal Processing Letters, vol. 13, no. 10, pp. 589-592, Oct. 2006.

[9] T. Aittomäki and V. Koivunen, "Iterative quadratic relaxation method for optimization of multiple radar waveforms," in IEEE International Conference on Acoustics, Speech and Signal Processing (ICASSP), Mar. 2016, pp. 3036-3040.

[10] S. U. Pillai, D. C. Youla, H. S. Oh, and J. R. Guerci, "Optimum transmit-receiver design in the presence of signal-dependent interference and channel noise," in Conference Record of the Thirty-Third Asilomar Conference on Signals, Systems, and Computers, Oct. 1999, vol. 2, pp. 870-875.

[11] J. R. Guerci, "Cognitive radar: A knowledge-aided fully adaptive approach," in 2010 IEEE Radar Conference, May 2010, pp. $1365-1370$.

[12] Harry L. Van Trees, Detection, Estimation, and Modulation Theory - Part III - Radar-Sonar Processing and Gaussian Signals in Noise, John Wiley \& Sons, 2001.

[13] G. San Antonio, D.R. Fuhrmann, and F.C. Robey, "MIMO radar ambiguity functions," IEEE Journal of Selected Topics in Signal Processing, vol. 1, no. 1, pp. 167-177, June 2007.

[14] D.H. Brandwood, "A complex gradient operator and its application in adaptive array theory," IEE Proceedings, vol. 130, no. 1, pp. 11-16, Feb. 1980, Parts F and H.

[15] J. Kennedy and R. Eberhart, "Particle swarm optimization," in IEEE International Conference on Neural Networks, Nov. 1995, vol. 4, pp. 1942-1948. 